\title{
BMJ Utilisation of primary total knee joint open replacements across socioeconomic status in the Barwon Statistical Division, Australia, 2006-2007: a cross-sectional study
}

\author{
Sharon Lee Brennan, ${ }^{1,2,3}$ Tyman Stanford, ${ }^{4}$ Anita E Wluka, ${ }^{5}$ Richard S Page, ${ }^{6}$ \\ Stephen E Graves, ${ }^{7}$ Mark A Kotowicz, ${ }^{1,2,8}$ Geoffrey C Nicholson,, ${ }^{1,9}$ \\ Julie A Pasco ${ }^{1,2}$
}

To cite: Brennan SL, Stanford T, Wluka AE, et al. Utilisation of primary total knee joint replacements across socioeconomic status in the Barwon Statistical Division, Australia, 20062007: a cross-sectional study. BMJ Open 2012;2: e001310. doi:10.1136/ bmjopen-2012-001310

- Prepublication history and additional material for this paper are available online. To view these files please visit the journal online (http://dx. doi.org/10.1136/bmjopen2012-001310).

Received 13 April 2012 Accepted 2 August 2012

This final article is available for use under the terms of the Creative Commons Attribution Non-Commercial 2.0 Licence; see http://bmjopen.bmj.com

For numbered affiliations see end of article

Correspondence to Dr Sharon L Brennan; sbrennan@unimelb.edu.au

\section{ABSTRACT}

Objectives: There are few Australian data that examine the association between total knee joint replacement (TKR) utilisation and socioeconomic status (SES). This study examined TKR surgeries with a diagnosis of osteoarthritis (OA) performed for residents of Barwon Statistical Division (BSD) for 2006-2007.

Design: Cross-sectional.

Setting: BSD, South-eastern Victoria, Australia Participants: All patients who underwent a TKR for OA, 2006-2007, and whose residential postcode was identified as within the BSD of Australia, and for whom SES data were available, were eligible for inclusion.

Primary outcome measure: Primary TKR data ascertained from the Australian Orthopaedic Association National Joint Replacement Registry. Residential addresses were matched with the Australian Bureau of Statistics census data, and the Index of Relative Socioeconomic Disadvantage was used to determine SES, categorised into quintiles whereby quintile 1 indicated the most disadvantaged and quintile 5 the least disadvantaged. Age-specific and sex-specific rates of TKR utilisation per 1000 personyears were reported for 10-year age bands.

Results: Females accounted for $62.7 \%$ of the 691 primary TKR surgeries performed during 2006-2007. The greatest utilisation rates of TKR in males was 7.6 observed in those aged $>79$ years, and in 10.2 in females observed in those aged 70-79 years. An increase in TKR was observed for males in SES quintile four compared to quintile 1 in which the lowest utilisation which was observed $(p=0.04)$. No differences were observed in females across SES quintiles.

Conclusions: Further investigation is warranted on a larger scale to examine the role that SES may play in TKR utilisation, and to determine whether any social disparities in TKR utilisation reflect health system biases or geographic differences.

\section{ARTICLE SUMMARY}

Article focus

- To determine whether total knee replacements (TKR) performed for arthritis were associated with socioeconomic status (SES).

- Using a comprehensive Australian registry of knee replacement surgeries, we assessed the association between quintiles of SES and TKR utilisation rates in males and females for 2006-2007.

Key messages

- Males in the most disadvantaged quintile appeared less likely to have a TKR compared to less disadvantaged males.

- No association between SES and TKR was observed for females.

- Highest rates of TKR utilisation for both sexes were observed in those aged $>79$ years, where increased TKR utilisation was observed for less disadvantaged males and females compared to most disadvantaged.

Strengths and limitations of this study

- TKR were ascertained from a comprehensive national registry relating to hip and knee replacements in Australia.

- The study region has been shown to be representative of the broader Australian population.

- We were unable to examine functional determinants of perceived need for TKR, or referral patterns for TKR.

- Sample size of $n=691$ may have limited our analyses.

\section{INTRODUCTION}

Osteoarthritis (OA) more commonly affects the knee joint, and is a leading cause of disability and reduced quality of life in the elderly. ${ }^{1-3}$ It is well documented that knee $\mathrm{OA}$ is more prevalent in females than in 
males, ${ }^{4}$ and that an increased incidence is observed in females, ${ }^{5}$ along with an increased progression of disease. ${ }^{6}$ Given that OA is not life threatening, primary total knee joint replacement (TKR) for end-stage OA is considered as elective surgery, regardless of sex. It is a cost-effective procedure for the intervention in advanced knee OA, and has shown high efficacy in relieving pain and increasing mobility. ${ }^{7} 8$ The number of TKR procedures performed Australia-wide was recently reported as in excess of 40000 , with a $3.7 \%$ increase from the previous year. $^{9}$

Studies from different countries present conflicting data of the association between joint replacement and socioeconomic status (SES). In England, prior to health reform of the National Health System (NHS), individuals of greater social disadvantage were less likely to undergo $\mathrm{TKR}^{10}{ }^{11}$ yet in Canada individuals of lower SES were more likely to have their need for TKR met than individuals of more advantage, ${ }^{12}$ and in Finland few differences between TKR across SES groups have been reported. ${ }^{13}$ Variations in TKR utilisations across SES have also been shown in other studies. ${ }^{14-19}$ Given that Australia has a readily accessible, universal health system, it would be expected that the uptake of TKR surgeries would not differ between socioeconomic groups, similar to the reduced disparities in TKR observed in England following NHS reforms to improve health equity. ${ }^{20}$ Compared to other countries, there are few Australian data examining SES and joint replacement; we have recently called for further data in an examination of hip joint replacements across SES, ${ }^{21}$ another study identified a lower rate of knee and hip joint replacements performed for socially disadvantaged individuals compared to less disadvantaged groups, ${ }^{22}$ and a third study identified reduced rates of joint replacement in Italian and Greek migrants to Australia compared to non-migrants. ${ }^{23}$ Given the limited Australian data, it is imperative to gain an improved understanding of the relationship between SES and TKR in order to inform health service provision; this could be considered long overdue.

The aim of this study was to determine whether TKR procedures, primarily performed for the elderly, were associated with SES in the Barwon Statistical Division (BSD), a region located in south-eastern Australia incorporating approximately 260000 residents (approximately $1.31 \%$ of the Australian population) in 2006, and comprising the Australian Electoral Commission Divisions of Corio, Corangamite (part) and Lalor (part). ${ }^{24}$ Using a comprehensive registry of knee replacement surgeries across Australia, we assessed the association between quintiles of SES and TKR utilisation rates in males and females for 2006-2007 for the BSD.

\section{MATERIALS AND METHODS TKR procedures}

Incident knee joint replacements that had been performed for OA during 2006-2007 for residents of the BSD were identified from the Australian Orthopaedic
Association National Joint Replacement Registry (AOANJRR). Implementation of the AOANJRR began in 1999 and was complete nationally by mid 2002. Since that time, all hospitals $(\sim 300)$, both public and private, undertaking arthroplasty procedures submit data to the registry. ${ }^{25}$ Data are matched and verified by crosslinking registry data with government separation data for all arthroplasty procedures. This verification process has established that the Registry receives information on more than $99 \%$ of all joint replacement operations. The registry monitors the performance and outcome of joint replacement procedures Australia-wide, and the data are provided via voluntary cooperation from all Australian hospitals performing joint replacement surgeries. ${ }^{26}$ The AOANJRR has been validated against health department unit record data using a sequential multilevel matching process and coupled with the retrieval of unreported procedures, making it the most complete set of joint replacement data in Australia. ${ }^{27}$

Primary TKR was defined as primary replacement of the tibiofemoral joint surfaces, and in some cases also the patellofemoral joint. Both conventional and resurfacing TKR were included. Primary partial TKR or revisions were not included within this analysis.

All patients that underwent a TKR during the study period, and whose residential postcode was identified as within the BSD of Australia were eligible for inclusion. Of the 753 primary TKR procedures fulfilling these criteria, 98\% ( $\mathrm{n}=738)$ had a diagnosis of OA. The remaining TKR were for rheumatoid arthritis $(n=10)$, tumour $(n=2)$, avascular necrosis $(n=2)$ or other reasons $(n=1)$.

\section{Socioeconomic status}

Postcodes were used to identify TKR procedures for those from the BSD, and the full residential address of each patient was then matched to the corresponding Australian Bureau of Statistics (ABS) Census Collection District, an area of approximately 250 households. ABS software was used to determine the Socio-Economic Indexes For Areas (SEIFA) value from the 2006 census for each patient; SEIFA values provide a single measure to rank the level of disadvantage at the area level, not of the individual subject. ${ }^{28}$ The SEIFA indices have been validated by analysts from ABS Regional Offices, and an external peer-review has been undertaken by a group of academic and policy research experts to examine the variables and methodology employed, ${ }^{29}$ and the principal components analysis employed by the ABS has been shown as reliable. ${ }^{29} 30$ The Index of Relative Socioeconomic Disadvantage (IRSD) incorporates variables that indicate people with (1) low incomes, (2) little or no training and (3) unskilled occupations, and was used for these analyses. Quintile 1 in the IRSD represented the most disadvantaged and quintile 5 represented the least disadvantage. SEIFA values were unavailable for 32 patients $(4.6 \%$ of the cases, $0.02 \%$ of the total population at risk), for reasons which included: fewer than 10 people residing in an area, fewer than 
5 people employed in an area, 5 or fewer occupied private dwellings in an area, or areas in which nonresponse to Census questions including occupation, labour force status, type of educational institution attending or non-qualifications exceeded $70 \%{ }^{29}$ The final number of patients included in this analysis totalled 691. The AOANJRR Data Review Committee, as a Federal Quality Assurance Activity under the Health Insurance Act of 1973, approved this study.

\section{Statistical analysis}

We calculated the rates of primary TKR for 10-year age strata for men and women; these were expressed as the number of surgeries per 1000 persons per year, based on each SES quintile.

Poisson regression was used to model the age-adjusted relative risk of primary TKR per unit time stratified by sex across SES quintiles. Given the interaction between SES and sex, the model was set up as:

$\log (\mathrm{N})=\log (\mathrm{PAR})+$ intercept + age group + SES + sex + SES x sex + error (where PAR=population attributable risk)

This model enables the examination of the rate of TKR (per unit of time) of N/PAR given the predictive variables of age group, SES and sex. In order to examine the rates of TKR within sex across SES groups (adjusting for age), we created post hoc comparisons of estimated N/PAR within sex across different SES quintiles $(\alpha=0.05)$. In order to examine the rates of TKR within sex across SES groups in the oldest age group of $>79$ years, we used the same model, excluding the age term. Two diagnostic plots were employed to check assumptions of the Poisson regression model; the Residual Quantile-Quantile Plot and the Residual versus Predicted Plot. The Residual Quantile-Quantile Plot showed that deviance was greater than 1 , suggesting that the model was overdispersed therefore a scale parameter was used to adjust the SDs. The Residual versus Predicated Plot presented with only a slight fanning of the residuals; however, apart from this there were no systematic trends. Analyses were performed using SAS V.9.2 (SAS Institute Inc, Cary, North Carolina, USA).

\section{RESULTS}

There were 691 TKR for OA, of which females accounted for $62.7 \%$. Table 1 presents the crude numbers and rates of TKR utilisation per 1000 person-years for each 10-year age group, sex and SES quintile. The greatest rate of TKR in the total group was observed in males aged $>79$ years (7.6/1000 person-years), in females aged 70-79 years (10.2/1000 person-years; table 1$)$.

Table 2 presents the sex-specific, age-adjusted relative risks of TKR utilisation. For females, there were no significant results at the $\alpha=0.05$ level from the post hoc comparisons. For males, individuals in quintile 4 had greater TKR utilisation compared to quintile 1 ; no other significant differences at the $\alpha=0.05$ level were observed in males. Compared to quintile 1 , there was an estimated increased rate of primary TKR for males in all SES groups; however, these were not-significant.

Table 1 Crude numbers and rates per 1000 person-years (based on population at risk in each socioeconomic quintile) of total knee joint replacement (TKR) replacements by age, sex and socioeconomic quintiles for 2006-2007

\begin{tabular}{|c|c|c|c|c|c|c|c|c|c|c|c|c|c|}
\hline & \multirow[b]{2}{*}{$\begin{array}{l}\text { Population } \\
\text { at risk }\end{array}$} & \multicolumn{2}{|l|}{ Total } & \multicolumn{2}{|c|}{$\begin{array}{l}\text { Quintile } \\
1^{*}\end{array}$} & \multicolumn{2}{|c|}{$\begin{array}{l}\text { Quintile } \\
2\end{array}$} & \multicolumn{2}{|c|}{$\begin{array}{l}\text { Quintile } \\
3\end{array}$} & \multicolumn{2}{|c|}{$\begin{array}{l}\text { Quintile } \\
4\end{array}$} & \multicolumn{2}{|c|}{$\begin{array}{l}\text { Quintile } \\
5\end{array}$} \\
\hline & & $\mathrm{n}=\mathrm{TKR} \boldsymbol{\mathrm { T }}$ & $\begin{array}{l}\text { Rate (per } 1000 \\
\text { person-years) }\end{array}$ & $\mathrm{n}=$ & Rate & $\mathbf{n}$ & Rate & $\mathbf{n}$ & Rate & $\mathbf{n}$ & Rate & $\mathbf{n}$ & Rate \\
\hline \multicolumn{14}{|c|}{ Males (years) } \\
\hline $20-29$ & 14727 & - & - & - & - & - & - & - & - & - & - & - & - \\
\hline $30-39$ & 16624 & - & - & - & - & - & - & - & - & - & - & - & - \\
\hline $40-49$ & 18192 & 3 & 0.1 & 1 & 0.2 & 1 & 0.2 & - & - & 1 & 0.1 & - & - \\
\hline $50-59$ & 17020 & 32 & 0.9 & 6 & 1.0 & 6 & 1.0 & 5 & 0.7 & 6 & 0.8 & 6 & 0.8 \\
\hline $60-69$ & 11767 & 106 & 4.5 & 8 & 1.8 & 25 & 5.3 & 19 & 4.0 & 21 & 4.4 & 26 & 5.5 \\
\hline $70-79$ & 8383 & 80 & 4.8 & 14 & 3.8 & 22 & 5.9 & 10 & 3.0 & 19 & 5.8 & 9 & 3.4 \\
\hline$>79$ & 4361 & 37 & 4.2 & 5 & 2.9 & 5 & 2.5 & 5 & 2.5 & 12 & 7.4 & 10 & 7.6 \\
\hline \multicolumn{14}{|l|}{$\begin{array}{l}\text { Females } \\
\text { (years) }\end{array}$} \\
\hline 20-29 & 14498 & 1 & 0.0 & 1 & 0.2 & - & - & - & - & - & - & - & - \\
\hline 30-39 & 17599 & 2 & 0.1 & 2 & 0.3 & - & - & - & - & - & - & - & - \\
\hline $40-49$ & 19367 & 3 & 0.1 & 1 & 0.1 & - & - & 1 & 0.1 & 1 & 0.1 & - & - \\
\hline 50-59 & 17653 & 58 & 1.6 & 12 & 1.9 & 11 & 1.7 & 7 & 0.9 & 14 & 1.9 & 7 & 1.0 \\
\hline $60-69$ & 12435 & 103 & 4.1 & 22 & 4.3 & 29 & 5.8 & 12 & 2.3 & 22 & 4.4 & 10 & 2.2 \\
\hline $70-79$ & 9872 & 191 & 9.7 & 42 & 9.4 & 46 & 10.2 & 36 & 8.6 & 35 & 9.7 & 26 & 8.8 \\
\hline$>79$ & 7320 & 75 & 5.1 & 9 & 3.0 & 12 & 3.5 & 20 & 5.9 & 16 & 5.7 & 17 & 8.2 \\
\hline
\end{tabular}

${ }^{*}$ Most disadvantaged.

$\lceil n=T K R$ in the total column includes primary TKR where Socio-Economic Indexes For Areas values were unavailable 
Table 2 Poisson model testing for relative risk (RR) of total knee joint replacement stratified by sex, and across socioeconomic quintiles. Quintile 1 (most disadvantaged) held as reference range for both sexes

\begin{tabular}{lllll}
\hline $\begin{array}{l}\text { Comparison with } \\
\text { SES quintile 1 }\end{array}$ & RR & $\begin{array}{l}\text { Lower } \\
\mathbf{9 5 \%} \mathbf{~ C l}\end{array}$ & $\begin{array}{l}\text { Upper } \\
\mathbf{9 5 \%} \mathbf{~ C l}\end{array}$ & $\begin{array}{l}\mathbf{p} \\
\text { Value }\end{array}$ \\
\hline Males & & & & \\
SES 2 & 1.68 & 0.98 & 2.87 & 0.06 \\
SES 3 & 1.12 & 0.62 & 2.00 & 0.71 \\
SES 4 & 1.76 & 1.03 & 3.01 & 0.04 \\
SES 5 & 1.70 & 0.98 & 2.95 & 0.06 \\
Females & & & & \\
SES 2 & 1.08 & 0.75 & 1.56 & 0.66 \\
SES 3 & 0.84 & 0.57 & 1.24 & 0.38 \\
SES 4 & 1.09 & 0.75 & 1.59 & 0.66 \\
SES 5 & 0.87 & 0.57 & 1.32 & 0.50 \\
\hline SES, socioeconomic status. & & &
\end{tabular}

\section{DISCUSSION}

Males in the most disadvantaged quintile appeared less likely to have a TKR compared to less disadvantaged males; however, this was only significant between SES quintiles 1 and 4. No association was observed for females. The highest rates of TKR utilisation for both sexes were observed in those aged $>79$ years; increased TKR utilisation was again observed for males in quintile 5 compared to quintile 1 , and this pattern was also observed for females.

Although it may be suggested that the need for a TKR does not discriminate between individuals based on their SES but instead on disease severity at end-stage OA, many lifestyle risk factors associated with OA severity, such as obesity, ${ }^{31}$ are predominantly associated with SES in both sexes. ${ }^{32} 33$ Therefore, greater end-stage disease may be more likely in the lower SES groups. However, our findings suggested the opposite situation, whereby increased TKR utilisation may actually exist for those of upper SES. We speculate that there may be various reasons for differences in the uptake of TKR surgeries between different socioeconomic quintiles, ${ }^{34} 35$ especially for the elderly, which may include patterns of referral; inequitable accessibility between the public and private health system ${ }^{36}$ or perhaps that a combination of these issues exist.

While healthcare is relatively equitable for all socioeconomic groups within Australia due to the availability of both private and public health systems, studies have suggested that social determinants of TKR utilisation may still include differential access to healthcare. ${ }^{23}{ }^{36}$ For instance, in a study of Italian and Greek migrants within Australia, similar numbers of non-Australian-born people underwent joint replacement in the public system compared to Australian-born individuals; however, differences between Australian-born and non-Australian-born patients were observed for those using the private health system. ${ }^{23}$ Given that we observed a significant difference between quintile 4 and the most disadvantaged SES quintile for males in the total population, and disparities between SES quintiles for females when limited to patients aged $>79$ years, we speculate public versus private healthcare usage may remain a determinant of TKR utilisation as populations age.

In addition to accessibility, differing social factors and beliefs in those from different socioeconomic quintiles may influence health-seeking behaviour, or the willingness to undergo a TKR procedure. Given that these issues are beyond the scope of this analysis, our discussion concerning the patient's decision to undertake or refuse TKR, and decision-making processes of the prescribing health professional, are purely speculative. Functional motivations have been suggested as a key driver for uptake of TKR where access to health care is equitable, with patients who self-report a higher range of movement (ROM) more likely to postpone surgery. ${ }^{37}$ It may be possible that individuals of lower SES have a greater threshold for functional limitations such as ROM, or perhaps given that individuals of lower SES are less likely to be physically active, ${ }^{32} 333839$ they are less likely to perform movements associated with full ROM.

Interestingly, it has been shown that TKR is more likely to be performed for males compared to females; ${ }^{40-42}$ however, we observed the rate of TKR for females to exceed that of males in the BSD. Although speculation, there may be two explanations for this observation. Higher body mass index (BMI) is a significant predictor for the development of knee OA; ${ }^{43-45}$ strong inverse relationship between BMI and SES is observed in a random sample of women shown as representative of the BSD population, and of the broader Australian population. ${ }^{32}$ However, this may not fully explain the differences between sexes in our sample, as a similar relationship between BMI and SES has also been observed in males in the same geographic region. ${ }^{33}$ Given this, we suggest that as knee OA is more common in women compared to men, ${ }^{46}$ our findings suggest that SES may play a role in exacerbation or mediation of disparities between TKR and sexes.

A cross-sectional association has been demonstrated in NHANES between the demands of occupational knee bending and knee $\mathrm{OA}$ in persons aged 55-64 years, ${ }^{47}$ and consistent with this was the Framingham study, which showed a relationship between occupational physical labour, knee bending and later OA, especially among males. ${ }^{48}$ These data are supported by a casecontrol study in the UK, which showed an increased risk of knee OA in subjects whose main job entailed more than $30 \mathrm{~min} /$ day of squatting or kneeling, or climbing more than 10 flights of stairs per day, or where the job entailed heavy lifting. ${ }^{49}$ These data suggest a role for at least one parameter of SES in knee OA. However, occupational knee bending is an unmeasured confounder in this analysis. Furthermore, it is unclear as to why the differences in TKR between upper and lower SES quintiles in men became more significant in the older age group, for whom physically demanding occupations may or may not have been a confounder, although given that the 
number of TKR performed does not indicate disease prevalence, but instead symptomatic disease, the need for TKR is in part a product of activity level as well as disease severity. Nevertheless, as a greater number of TKR are performed in older individuals compared to their younger counterparts, this observation may be of little consequence unless there was a concomitant increase or decrease in the magnitude of the effect, which did not appear to be the case in this study.

One strength of this study was that the TKR were ascertained from a comprehensive national registry that has been validated against health department unit record data using a sequential multilevel matching process, and, coupled with the retrieval of unreported records, is the most complete set of data relating to hip and knee replacement in Australia. Of the TKR cases identified for 2006-2007 in the BSD, 6\% could not be coded for SES, which may have influenced the association with SES. However, given that the spread of these patients was relatively equal between sexes ( $49 \%$ male), and represented equal proportions of the population at risk for each sex (both $0.02 \%$ ), any potential disparity would be non-differential. The $\mathrm{BSD}$ region has been shown to be representative of the broader Australian population and thus provides an excellent location for epidemiological research. However, these observations cannot be assumed to exist in other geographic regions of Australia, or relate to the country as a whole. We were unable to examine functional determinants of perceived need for TKR, uptake and/or referral patterns for TKR, or lifestyle risk factors in our population. Furthermore, the AOANJRR database does not collect information regarding comorbidities, medications or other factors that may influence associations between SES and TKR, thus we were unable to account for these factors in our analyses. Given that sample size limited our analyses, it is imperative that this analysis be performed on a larger scale, to determine whether SES is associated with TKR for the broader Australian population.

Further investigation is warranted on a larger scale to investigate the role that SES may play in TKR utilisation, and whether this may be related to differences in referral for TKR, differences in OA prevalence or in healthseeking behaviour.

\footnotetext{
Author affiliations

${ }^{1}$ NorthWest Academic Centre, Department of Medicine, The University of Melbourne, St Albans, Victoria, Australia

${ }^{2}$ Barwon Epidemiology and Biostatistics Unit, School of Medicine, Deakin University, Geelong, Australia

${ }^{3}$ Australian Institute of Musculo-Skeletal Science, NorthWest Academic Centre, The University of Melbourne, St Albans, Victoria, Australia

${ }^{4}$ Data Management and Analysis Centre, Discipline of Public Health, University of Adelaide, Adelaide, Australia

${ }^{5}$ Department of Epidemiology and Preventive Medicine, School of Preventive Medicine, Monash University, Melbourne, Australia

${ }^{6}$ Barwon Orthopaedic Research Unit, Barwon Health, Geelong, Australia

${ }^{7}$ Australian Orthopaedic Association Joint Replacement Registry, Adelaide, Australia

${ }^{8}$ Department of Endocrinology and Diabetes, Barwon Health, Geelong, Australia
}

${ }^{9}$ Rural Clinical School, School of Medicine, The University of Queensland, Toowoomba, Australia

Acknowledgements We thank Ann Tomkins, and the AOANJRR team for assisting with data access.

Contributors All authors participated in the study design. SLB drafted the manuscript. TS performed the statistical analysis and SEG and SLB supervised the analysis. SLB, MAK, GCN, AEW, RSP and JAP guided and reviewed the manuscript. All authors read and approved the final manuscript.

Funding SLB is supported by a National Health and Medical Research Council (NHMRC) Early Career Fellowship (1012472, 2011-14). AEW is the recipient of NHMRC Career Development Award (545876).

Competing interests SLB, TS, MAK, GCN, and JAP have no competing interests. AEW is a Committee member of the Scientific Programme and Research Committee, Australian Rheumatology Association, and is on the Advisory Board of the Australian Rheumatology Association Research Trust Scientific Advisory Board. RSP has received research support from Ascension Orthopaedics, and financial support from Synthes, and De Ply Education for Fellowship Position. RSP is a Committee member of the Australian Orthopaedic Association National Joint Replacement Registry (AOANJRR), from which the dataset used for this analysis were extracted, and is an Executive of the Shoulder and Elbow Society. SEG is the director of the AOANJRR, from which the dataset used for this analysis was extracted.

Provenance and peer review Not commissioned; externally peer reviewed. Data sharing statement No additional data are available.

\section{REFERENCES}

1. Yelin $\mathrm{E}$, Lubeck $\mathrm{D}$, Holman $\mathrm{H}$, et al. The impact of rheumatoid arthritis and osteoarthritis: the activities of patients with rheumatoid arthritis and osteoarthritis compared to controls. J Rheum 1987; 14:710-17.

2. Guccione A, Felson DT, Anderson JJ, et al. The effects of specific medical conditions on the functional limitations of elders in the Framingham study. Am J Public Health 1994;84:351-8.

3. Hubert $\mathrm{H}$, Bloch DA, Fries JF. Risk factors for physical disability in an ageing cohort: the NHANES I follow-up survey. J Rheum 1993;30:480-8.

4. Blagojevic M, Jinks C, Jeffery A, et al. Risk factors for onset of osteoarthritis of the knee in older adults: a systematic review and meta-analysis. Osteoarthrit Cart 2010;18:24-33.

5. Schouten J, van den Ouweland FA, Valkenburg HA. A 12 year follow up study in the general population on prognostic factors of cartilage loss in osteoarthritis of the knee. Ann Rheum Dis 1992;51:932-7.

6. Felson D, Zhang Y, Hannan MT, et al. Risk factors for incident radiographic knee osteoarthritis in the elderly: the Framingham study. Arthritis Rheum 1997;40:728-33.

7. Walker DJ, Heslop PS, Chandler C, et al. Measured ambulation and self-reported health status following total joint replacement for the osteoarthritic knee. Rheumatology (Oxford) 2002;41:755-8.

8. Chang RW, Pellisier JM, Hazen GB. A cost-effectiveness analysis of total hip arthroplasty for osteoarthritis of the hip. JAMA 1996; 275 : 858-65.

9. Australian Orthopaedic Association National Joint Replacement Registry. Analysis of State and Territory Health data: all arthroplasty. Adelaide: AOANJRR 2009:1-23.

10. Dixon T, Shaw M, Ebrahim S, et al. Trends in hip and knee joint replacement: socioeconomic inequalities and projections of need. Ann Rheum Dis 2004;63:825-30.

11. Yong PFK, Milner PC, Payne JN, et al. Inequalities in access to knee joint replacements for people in need. Ann Rheum Dis 2004:63:1483-9.

12. Hawker GA, Wright JG, Glazier RH, et al. The effect of education and income on need and willingness to undergo total joint arthroplasty. Arthrit Rheum 2002;46:3331-9.

13. Westert GP, Smits JPJM, Polder JJ, et al. Community income and surgical rates in the Netherlands. J Epidemiol Comm Health 2003;57:519-22.

14. Judge A, Welton NJ, Sandhu J, et al. Geographical variation in the provision of elective primary hip and knee replacement: the role of socio-demographic, hospital and distance variables. J Pub Health 2009;31:413-22. 
15. Cookson R, Dusheiko M, Hardman G. Socioeconomic inequality in small area use of elective total hip replacement in the English National Health Service in 1991 and 2001. J Health Serv Res Policy 2007;12(Suppl 1):10-17.

16. Majeed A, Eliahoo J, Bardsley M, et al. Variation in coronary artery bypass grafting, angioplasty, cataract surgery, and hip replacement rates among primary care groups in London: association with population and practice characteristics. J Pub Health Med 2002;24:21-6.

17. Steel N, Melzer D, Gardener E, et al. Need for and receipt of hip and knee replacement-a national population survey. Rheumatol 2006;45:1437-41.

18. Melzer D, Guralnik JM, Brock D. Prevalence and distribution of hip and knee joint replacements and hip implants in older Americans by the end of life. Aging Clin Exp Res 2003;15:60-6.

19. Agabiti N, Picciotto S, Cesaroni G, et al. The influence of socioeconomic status on utilization and outcomes of elective total hip replacement: a multicity population-based longitudinal study. Int J Quality in Health Care 2007;19:37-44.

20. Cooper ZN, McGuire A, Jones S, et al. Equity, waiting times, and NHS reforms: retrospective study. BMJ 2009;339:673-5.

21. Brennan SL, Stanford T, Wluka AE, et al. Cross-sectional analysis of association between socioeconomic status and utilization of primary total hip joint replacements 2006-7: Australian Orthopaedic Association National Joint Replacement Registry. BMC Musculoskelet Disord 2012;13:63

22. Dixon T, Urquhart DM, Berry $\mathrm{P}$, et al. Variation in rates of hip and knee joint replacement in Australia based on socio-economic status, geographical locality, birthplace and indigenous status. ANZ J Surg 2011;81:26-31.

23. Wang $Y$, Simpson JA, Wluka AE, et al. Reduced rates of primary joint replacement for osteoarthritis in Italian and Greek migrants to Australia: the Melbourne Collaborative Cohort Study. Arthrit Res Ther 2009; 11:R86.

24. Pasco JA, Nicholson GC, Kotowicz MA. Cohort profile: Geelong Osteoporosis Study. Int J Epidemiol 2011;12:1-11.

25. Australian Orthopaedic Association National Joint Replacement Registry. Australian Orthopaedic Association National Joint Replacement Registry. Annual Report. Adelaide: AOANJRR, 2007.

26. Graves SE, Davidson D, Ingerson L, et al. The Australian Orthopaedic Association National Joint Replacement Registry. Med J Aust 2004;180:S31-4.

27. Australian Orthopaedic Association National Joint Replacement Registry. Demographics of hip and knee arthroplasty. Adelaide: AOANJRR 2009:1-33.

28. An introduction to socio-economic indexes for areas (SEIFA). In, vol ABS Cat No 2039.0. Canberra: Australian Bureau of Statistics, 2006.

29. Australian Bureau of Statistics. Socio-economic Indices for Areas (SEIFA)—-technical paper. Canberra: ABS, 2006.

30. Dudzinski M, Norris J, Chmura J, et al. Repeatability of principal components in samples: normal and non-normal data sets compared. Multivariate Behav Res 1975;10:109-17.

31. Brennan SL, Cicuttini FM, Pasco JA, et al. Does an increase in body mass index over 10 years affect knee structure in a population-based cohort study of adult women? Arthrit Res Ther 2010;12:R139.

32. Brennan SL, Henry MJ, Nicholson GC, et al. Socioeconomic status and risk factors for obesity and metabolic disorders in a population-based sample of adult females. Prev Med 2009;49:165-71.

33. Brennan SL, Henry MJ, Nicholson GC, et al. Socioeconomic status, obesity and lifestyle in men: the Geelong Osteoporosis Study. $J$ Men's Health 2010;7:31-41.

34. Sanders C, Donovan JL, Dieppe PA. Unmet need for joint replacement: a qualitative investigation of barriers to treatment among individuals with severe pain and disability of the hip and knee. Rheumatology 2004;43:353-7.

35. Hawker GA. Who, when, and why total joint replacement surgery? The patient's perspective. Curr Opinion Rheumatol 2006;18:526-30.

36. Skinner J, Weinstein JN, Sporer SM, et al. Racial, ethnic, and geographic disparities in rates of knee arthroscopy among Medicare patients. N Engl J Med 2003;349:1350-9.

37. Zeni JA, Axe MJ, Snyder-Mackler L. Clinical predictors of elective total joint replacement in persons with end-stage knee osteoarthritis. BMC Musculoskelet Disord 2010;11:86.

38. Singh GK. Independent and joint effects of socioeconomic, behavioral, and neighborhood characteristics on physical inactivity and activity levels among US children and adolescents. J Comm Health 2008;33:206-16.

39. Crespo CJ, Ainsworth BE, Keteyian SH, et al. Prevalence of physical inactivity and its relation to social class in US adults: results from the Third National Health and Nutrition Examination Survey, 1988-1994. Med Sci Sports Exerc 1999;31:1821-7.

40. Dieppe P, Basler HD, Chard J, et al. Knee replacement surgery for osteoarthritis: effectiveness, practice variations, indications and possible determinants of utilization. Rheumatology (Oxford) 1999;8:3-83.

41. Mancuso CA, Ranawat CS, Esdaile JM, et al. Indications for total hip and knee arthroplasties. Results of orthopaedic surveys.

$J$ Arthroplast 1996;11:4-46.

42. Borkhoff CM, Hawker GA, Kreder HJ, et al. Patients' gender affected physicians' clinical decisions when presented with standardized patients but not for matching paper patients. J Clin Epidemiol 2009;62:527-41.

43. Felson D. Obesity and osteoarthritis of the knee. Bull Rheum Dis 1992;41:6-7.

44. Hartz AJ, Fischer ME, Bril G, et al. The association of obesity with joint pain and osteoarthritis in the HANES data. J Chronic Dis 1986;39:311-19.

45. Lievense A, Bierma-Zeinstra SMA, Vergahen AP, et al. Influence of obesity on the development of osteoarthritis of the hip: a systematic review. Rheumatology 2002;41:1155-62.

46. Tsai C, Liu TK. Osteoarthritis in women: its relationship to estrogen and current trends. Life Sci 1992;50:1737-44.

47. Andersson J, Felson DT. Factors associated with osteoarthritis of the knee in the NHANES I survey: evidence for an association with overweight, race and physical demands of work. Am J Epidemiol 1988;128:179-89.

48. Felson D, Hannan MT, Naimark A, et al. Occupational physical demands, knee bending, and knee osteoarthritis: results from the Framingham study. J Rheumatol 1991;18:1587-92.

49. Cooper C, McAlindon T, Coggon D, et al. Occupational activity and osteoarthritis of the knee. Ann Rheum Dis 1994;53:90-3. 